\title{
IMPORTÂNCIA DA CRIPTOSPORIDIOSE COMO ZOONOSE
}

\author{
André Luiz Baptista Galvão, Edna Gómez Ortiz¹, Guadalupe Sampaio Ferreira1, \\ Amanda Leal de Vasconcellos ${ }^{1}$, Manuela Cristina Vieira ${ }^{1}$, Kátia Denise Saraiva \\ Bresciani2 $^{2}$
}

1 FCAV - Unesp Jaboticabal - andrelgalvao@hotmail.com

2 FMV - Unesp Araçatuba

\begin{abstract}
RESUMO: Os protozoários do gênero Cryptosporidium sp. são parasitos que completam seu ciclo biológico na superfície de células epiteliais dos tratos respiratório, gastrointestinal e urinário de mamíferos, aves, répteis e peixes. A criptosporidiose é uma enfermidade que quando ocorre na forma de infecção natural, geralmente se apresenta assintomática ou com alterações gastrointestinais. Considerada uma zoonose, pois os animais quando infectados, principalmente os bovinos, constituem em fonte de infecção para o ambiente e para o homem, uma vez que eliminam grandes quantidades de oocistos em suas fezes. A sintomatologia quando presente é caracterizada por diarréia aquosa, desidratação, dores abdominais, perda de peso e morte, principalmente em indivíduos imunossuprimidos, e notadamente em paciente com vírus da imunodeficiência humana (HIV) e em crianças. Métodos terapêuticos eficazes no combate deste agente para os animais e para o homem, até o presente momento não foram estabelecidos, sendo aplicado o tratamento de suporte. Desta forma, trata-se de uma doença emergente, e a organização mundial de saúde a colocou na lista das doenças negligenciadas. Medidas de profilaxia são recomendadas como saneamento básico, utilização de métodos apropriados para a inativação dos oocistos e garantia de higiene pessoal constituem táticas para se minimizar a disseminação do Cryptosporidium. A presente revisão literária teve como objetivo descrever a importância da criptosporidiose em saúde pública.
\end{abstract}

Palavras-chave: bovinos; cão; Cryptosporidium; gato

\section{IMPORTANCE OF CRYPTOSPORIDIOSIS AS ZOONOSIS}

\begin{abstract}
The protozoa of the genus Cryptosporidium sp. are parasites that complete their life cycle on the surface of epithelial cells of the respiratory, gastrointestinal and urinary tracts of mammals, birds, reptiles and fish. Affected animals generally are asymptomatic but gastrointestinal disorders sometimes can be present. Criptoporidiosis is considered a zoonosis. Infected animals, especially cattle, are a source of infection for the environment and humans, because they eliminate large numbers of oocysts in their feces. The symptoms when present is characterized by watery diarrhea, dehydration, abdominal pain, weight loss and death, especially in immunosuppressed individuals, and especially in patients with human immunodeficiency virus (HIV) and children. Therapeutic methods effective to eliminate this agent in animals and humans has not been developed but it is necessary to applied support treatment. Criptosporidiosis is considered by the World Health Organization as an emerging disease. Basic sanitation, use of appropriate methods for the inactivation of oocysts and security of personal hygiene are recommended as Prophylactic methods to minimize the spread of Cryptosporidium. This literature review was aimed at describing the importance of cryptosporidiosis in public health.
\end{abstract}

Key Words: bovine; cat; Cryptosporidium; dog 


\section{INTRODUÇÃO}

O Cryptosporidium spp. trata-se de um enteroparasito rotineiramente encontrado nas fezes de animais domésticos e no ambiente (Fiúza et al., 2008). Atualmente está estabelecido que o gênero Cryptosporidium pertence ao Filo Apicomplexa, Classe Sporozoae, subclasse Coccidia, ordem Eucoccidiida, sub-ordem Eimeriina, Família Cryptosporidiidae e gênero Cryptosporidium (Plutzer e Karanis, 2009).

Protozoários do gênero Cryptosporidium compreendem em 21 espécies e mais de 61 genótipos, com grande diversidade genética, de caráter cosmopolita e que podem infectar cerca de 150 espécies de animais (Smith e Nichols, 2010), logo foram reportadas as seguintes espécies e seus respectivos hospedeiros como demonstrado na Tabela 01.

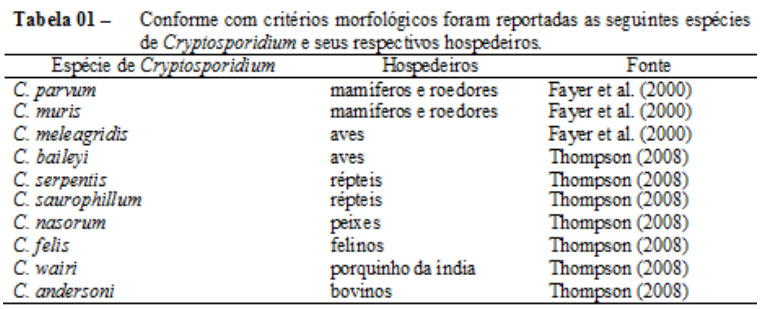

A criptosporidiose, zoonose com ampla distribuição geográfica, vem sendo estudada, particularmente devido a sua importância em saúde pública, pois são comuns os episódios diarréicos em crianças e adultos imunocompetentes, evoluindo para a cura espontânea (Thompson, 2008). Contudo, este protozoário pode ocasionar enterite grave em indivíduos imunocomprometidos, associada a desidratação e desnutrição, muitas vezes fatal, em portadores do vírus da imunodeficiência humana (HIV) (Rossit et al., 2007).

Animais de produção, bem como os cães e os gatos representam potenciais riscos para a saúde, uma vez que quando infectados podem agir como disseminadores de formas evolutivas parasitarias deste parasito no ambiente (Overgaauw et al., 2009).

O reconhecimento do Cryptosporidium spp. como um importante patógeno pela Organização Mundial de Saúde pôs em evidência o impacto da infecção por este parasito. Suas implicações na saúde da criança em termos de infecção e desenvolvimento, a continuação da epidemia de infecção pelo vírus do HIV e ao aumento do número de indivíduos imunossuprimidos, justamente com limitadas opções em tratamento, vêm mantendo este parasito em destaque (Chalmers e Davies, 2010). Desse modo, apresentamos esta revisão literária com o objetivo em descrever a importância da criptosporidiose em saúde pública, como potencial agente zoonótico.

\section{DESENVOLVIMENTO}

\section{Aspectos Criptosporidiose: \\ zoonóticos}

da

O Cryptosporidium é transmitido entre indivíduos por meio de oocistos que são eliminados na forma infectante nas fezes, sendo as principais vias de transmissão 0 contato direto (interpessoal) ou indiretamente pela ingestão de alimentos ou água contaminada. A dose mínima infectante varia de 9 a 1042 oocistos dependendo da amostra (Fayer et al., 2000). Esses oocistos mantêm sua infectividade por vários meses, principalmente sob condições ambientais favoráveis (Sunnotel et al., 2006).

Segundo Fayer et al. (2000) a importância da veiculação hídrica da criptosporidiose foi reconhecida a partir de um grande surto de Milwaukee, em 1993, neste surto 403.000 pessoas foram infectadas, e dessas, 4.400 foram hospitalizadas, ocorrendo 100 óbitos, sendo 69 óbitos de indivíduos 
portadores do vírus do HIV. Conforme Costa et al. (2006) este surto teve como custo, o total de 90,2 milhões de dólares, com medidas terapêuticas e profiláticas no controle da doença.

O Cryptosporidium tem sido detectado em esgotos ou em redes de fornecimento de água, desse modo a água, quando contaminada, pode atuar como fonte de infecção deste parasito para o ser humano (Olson et al., 2004). Nos alimentos, oocistos de Cryptosporidium sp. também foram encontrados como demonstrado na Tabela 02.

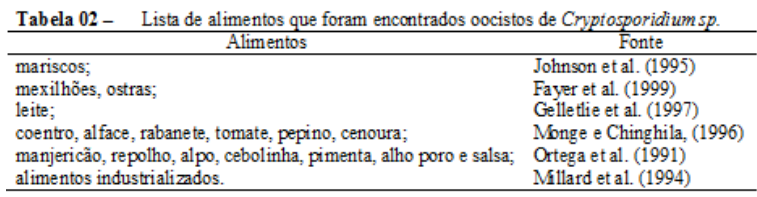

Conforme Smith et al. (2008) fatores podem ser atribuídos a ocorrência da criptosporidiose humana como: à elevada quantidade de oocistos esporulados, infectantes, excretados pelos hospedeiros; a pequena dose de formas evolutivas requeridas para a infecção (menos de 10 oocistos); a baixa especificidade pelos mamíferos e o fato dos oocistos serem resistentes à inativação por diversos desinfetantes em condições ambientais

Chacin-Bonilla (2001) descreveu que a criptosporidiose humana foi anteriormente atribuída somente aos genótipos humanos e bovinos, entretanto, outras espécies como $C$. felis, $C$. canis e $C$. meleagridis podem ser causadores da infecção no homem.

Em relação aos cães e gatos, quando infectados pelo Cryptosporidium spp. podem manifestar uma infecção assintomática ou com sinais clínicos de diarréia crônica ou intermitente (Moura et al. 2009).

Figueiredo et al. (2004) analisaram 269 amostras de fezes de cães saudáveis, de áreas urbanas e rurais dos municípios de Lavras e
Viçosa, Minas Gerais, Brasil, pelo método de Elisa direto e detectaram $1,85 \%$ (5/269) com positividade para Cryptosporidium parvum. Ederli et al. (2008) estudaram 200 amostras fecais de cães domiciliados na cidade de Campos dos Goytacazes, RJ, Brasil, e constataram a positividade de $45 \%$ (90/200) das amostras para Cryptosporidium, pela técnica de ZielhNeelsen modificada. No município de Lages, SC, Brasil, Moura et al. (2009) avaliaram 200 amostras de fezes de cães, por esfregaços pela técnica modificada de coloração de ZiehlNeelsen, e encontraram a ocorrência de 4\% (8/200) de Cryptosporidium sp.. Entre estes animais, os que possuíam dieta caseira e pertenciam a domicílios com acesso a terra e/ou a grama apresentaram maior positividade.

Em gatos, estudos realizados por Thompson et al. (2008) mostraram que o $C$. felis acomete mais rotineiramente os gatos, podendo ser encontrado em outras espécies de animais e em seres humanos.

Na Austrália, Palmer et al. (2008) utilizando a técnica de PCR, constataram a ocorrência de 2,44\% (26/1063) de amostras positivas para Cryptosporidium em felinos, pela caracterização molecular foi determinada exclusivamente a presença de $C$. felis nos gatos examinados. Huber et al. (2002) estudaram 48 amostras de fezes de gatos adultos, encontraram a positividade de 12,5\% (6/48) para Cryptosporidium sp.. Arai et al. (1990) encontraram uma positividade de 3,8\% (23/608) de Cryptosporidium em amostras fecais de gatos em Tóquio no Japão. Em exames post mortem realizados por Mtambo et al. (1991) revelaram que $8,1 \%(19 / 235)$ dos gatos na área de Glasgow estavam infectados por este coccídeo.

Segundo Caccio et al. (2005) o C. hominis e o $C$. parvum genótipo bovino são as espécies mais detectadas no ser 
humano. O C. parvum genótipo bovino infecta em condições naturais o homem e outros ruminantes, e em condições experimentais camundongos, ratos e cães.

Em ruminantes, na região de Araçatuba-SP, Brasil, Feitosa et al. (2004) analisaram 459 amostras fecais de bezerros com até 30 dias pelo método de Sheather e evidenciaram maior ocorrência de $C$. parvum em bezerros de 8 a 14 dias de idade e menor taxa, em animais mais velhos com 22 a 30 dias de vida. Kuczynska e Shelton (1999) consideraram bezerros leiteiros e de corte, importantes reservatórios de oocistos, devido ao elevado número de oocistos por eles excretados quando infectados. Almeida et al. (2008) verificaram pelas técnicas de Ritchie e de Ziehl-Neelsen modificadas, incidência de $61 \%(61 / 100)$ de positividade de bezerros do norte do estado do Rio de Janeiro, Brasil.

Em ovinos, um estudo realizado em 21 propriedades por Féres (2008), constatou a ocorrência de 6,73\% (31/460) de Cryptosporidium spp., no município de Araçatuba-SP, Brasil, sendo a principal espécie e genótipo envolvido o Cryptosporidium parvum e genótipo cervídeo, as amostras foram identificadas por PCR. Tembue et al. (2006) detectaram ocorrência de 3,7\% (3/81) de Cryptosporidium spp. pela técnica de Kinyon. em 22 propriedades do município de Ibirim-PE, Brasil.

Este protozoário já foi identificado em outros animais de produção, como nos suínos. Johnson et al. (2008), na Austrália, registraram ocorrência de $32,7 \%$ (51/1256) de Cryptosporidium spp. em leitões. Fiúza et al. (2008), no Rio de Janeiro, observaram que $40 \%$ $(20 / 50)$ dos suínos das granjas familiares e 29,3\% (12/53) das granjas tecnificadas apresentavam eliminação de oocistos de Cryptosporidium spp. nas fezes, pelas técnica de sedimentação e Ziehl-Neelsen modificada. Matos (2009) verificaram pela técnica de Kinyon 4,7\% (5/107) de positividade para Cryptosporidium spp. em leitões de até 45 dias de idade no município de Araçatuba-SP, Brasil.

A presença de $C$. parvum já foi detectada em animais aquáticos, como em leões marinhos da Califórnia (Zalophus californianus), por Deng et al. (2000) e em exemplares de focasanciadas (Phoca híspida) por HughesHanks et al. (2005).

Dietz et al. (2000) afirmaram que a contaminação por $C$. parvum nos Estados Unidos da América em seres humanos está associada à ingestão de água e de alimentos contaminados, bem como o contato direto com animais. Machado et al. (2009) descreveram que a liberação de oocistos presentes nas fezes dos hospedeiros infectados pode acarretar na contaminação de águas superficiais e reservatórios, os quais têm sido reconhecidos como fontes veiculadores do patógeno pela possibilidade de atingir um intenso contingente de hospedeiros, principalmente o homem. Os seres humanos com o sistema imunológico debilitado ou não, podem infectar-se com o C. parvum, C. meleagridis, $C$. canis, C. felis, C. suis, $C$. muris e Cryptosporidium genótipos de cervídeo e de macacos. Entre estas espécies o C. meleagridis, originalmente descrito em aves, tem apresentado um aumento de detecção em pessoas imunocompetentes sendo considerado hoje como um protozoário emergente e responsável por $11 \%$ das infecções em indivíduos imunocomprometidos no Reino Unido (Caccio et al., 2005).

Desta forma, muitos pesquisadores têm sugerido que todas as espécies de Cryptosporidium possam ser consideradas como potencialmente infectantes para o ser humano (Feltus et al., 2006). Garlipp et al. (1995) estudaram 188 amostras de fezes provenientes de 111 pacientes com 
diarréia, atendidos no Hospital das Clínicas da Universidade Estadual de Campinas, para detecção de Cryptosporidium spp, empregando a técnica de Ziehl-Nielsen modificada. Destes 111 pacientes, 83\% (92/111) eram HIV positivos. O Cryptosporidium spp foi observado em 18\% (16/111) de todos os pacientes, sendo que destes, 90\% (14/16) eram HIV positivos.

Araújo et al. (2007) estudaram 29 amostras de fezes de pacientes com HIV, utilizando o exame parasitológico, em 15 amostras foram identificadas oocistos de Cryptosporidium spp.. Posteriormente estas amostras foram submetidas a caracterização genotípica por meio de PCR-RFLP, sendo encontrado, dois isolados de $C$. hominis, uma amostra de $C$. parvum e ainda duas amostras de $C$. meleagridis, demonstrando a circulação o coccídeo no ambiente estudado, representando risco ao homem e aos animais.

Lucca et al. (2009) descreveram a frequência relativa de diversas espécies de Cryptosporidium em amostras de fezes de humanos da cidade de São Paulo, Brasil, obtidas de janeiro a julho de 2007, as análises de sequência de produtos de nested PCR, revelaram $63 \%(17 / 27)$ isolados do $C$. hominis, $14,8 \%(4 / 27)$ de C. parvum, $18,5 \%(5 / 27)$ de $C$. felis e $3,7 \%$ (1/27) de $C$. canis, sugerindo a transmissão zoonótica de criptosporidiose.

A facilidade de transmissão desse parasito para o ser humano, aliada ao fato da inexistência de um tratamento efetivo para a criptosporidiose, faz com que esse parasito continue a ser uma importante causa de morbidade e mortalidade, principalmente em indivíduos imunossuprimidos e crianças em países em desenvolvimento (Leav et al., 2003). Conforme Bolter-Bittzer et al. (2007), pessoas desnutridas, idosos e indivíduos imunocomprometidos, apresentam alto risco de desenvolver a diarréia persistente, quando expostos ao protozoário Cryptosporidium spp. Estudos realizados por Chieff et al. (1997) em indivíduos humanos imunocomprometidos com doença renal crônica e/ ou hipertensos foi observado a ocorrência da infecção por C. parvum em $34,8 \%(18 / 23)$ nos pacientes submetidos ao transplante renal, $25,0 \%$ $(8 / 32)$ nos pacientes submetido a hemodiálise e $17,4 \%$ (5/27) pacientes pertencentes ao grupo controle com hipertensão arterial sistêmica.

No Brasil o protozoário representa causa de morbidade e mortalidade de crianças de 0 a 5 anos de idade e em pacientes portadores do vírus do HIV (Oshiro et al., 2000). De acordo com Capuano et al. (2006) a população infantil corresponde ao grupo de mais alto risco, devido ao hábito de permanecer em contato com o solo e aos distúrbios de perversão do apetite como a geofagia. Segundo OliveiraSilva et al. (2007) o status imunológico do hospedeiro define o curso clínico da doença, pois quando um indivíduo apresenta 0 número de células de defesa CD4 inferior que 200 células $/ \mathrm{mm}^{3}$, ele apresentará uma infecção severa e persistente.

Nascimento et al. (2009) utilizando a técnica de Ziehl-Neelsen modificada, precedida pela técnica de sedimentação espontânea, analisaram amostras fecais de crianças de uma creche em Várzea, Recife, e reportaram $32,4 \%$ (59/182) de amostras positivas para Cryptosporidium spp.

Carvalho-Almeida et al. (2006) descreveram a ocorrência de 20,3\% (13/64) de oocistos de Cryptosporidium nas fezes de 64 crianças estudadas, sem diarréia, em uma escola de educação infantil da cidade São Paulo, Brasil. Oshiro et al. (2000) reportaram a ocorrência do Cryptosporidium na zona urbana de Campo Grande, Mato Grosso do Sul, Brasil, de 1,1\% em crianças menores de 5 anos. Pereira et al. (2002) 
relataram a ocorrência de 18,7\% C. parvum em crianças menores que 10 anos na cidade de Goiânia, Goiás, Brasil. Moitinho et al. (1997) analisaram 136 amostras de fezes de crianças de Maringá, Paraná, Brasil, e reportaram $6,6 \%(9 / 136)$ das amostras de fezes com oocistos de Cryptosporidium sp., utilizando o método de sedimentação em formol éter e posterior coloração em esfregaços. Silva et al. (1994) estudaram as amostras de fezes de 156 lactantes no Hospital geral do Instituto Materno-Infantil de Pernambuco, Brasil, e encontraram $6,4 \% \quad(10 / 156)$ nas amostras, o Cryptosporidium sp., neste estudo foi realizado a técnica de flutuação em sacarose, segundo a técnica de Sheater.

O contagio inter- pessoal é um fator epidemiológico importante de criptosporidiose sendo comum em creches. Nestas instituições, a ocorrência varia entre $1,0 \%$ e $20,0 \%$ e é apontado como uma das causas mais freqüentes de diarréia no Brasil, com registros de surtos epidêmicos relacionados ao $C$. hominis (Gonçalves et al., 2006).

Estudos realizados por Chieff et al. (1997) em indivíduos humanos imunocomprometidos com doença renal crônica foi observada a ocorrência da infecção por Cryptosporidium parvum em $34,8 \% \quad(18 / 23)$ nos pacientes submetidos a transplante renal, $25,0 \%$ (8/32) nos pacientes em hemodiálise e $17,4 \%(5 / 27)$ pacientes pertencentes ao grupo controle com hipertensão arterial sistêmica.

\section{Medidas de profilaxia e controle:}

Segundo Fayer et al. (2000) a ingestão de água contaminada, mostrase a principal via de infecção humana, pelo menos 165 surtos epidêmicos foram registrados nos últimos anos devido ao consumo de água contaminada com C. parvum.
Em virtude da ampla distribuição das diferentes espécies de Cryptosporidium, torna-se de fundamental importância 0 controle ambiental, por meio de adoção de práticas para prevenir a poluição de rios por excretas de animais (Graczyk et al., 2000), logo, o incentivo à adequação das estruturas de saneamento, a proteção dos mananciais, educação e orientação sobre descargas de resíduos por embarcações durante atividades náuticas, se tornam medidas recomendadas (Spósito Filha; Oliveira, 2009).

Nos alimentos oferecidos aos homens e aos animais, deve-se evitar a presença dos oocistos, logo, incentivar normas de higiene pessoal entre os tratadores e técnicos envolvidos no manejo de animais, são condutas necessárias (Spósito Filha; Oliveira, 2009).

Os animais especialmente bezerros leiteiros, muitas vezes se tornam infectados com C. parvum, e eliminam grandes quantidades de oocistos em suas fezes, por um período prolongado, contaminando o ambiente e tornando a infecção viável ao homem. Uma alternativa que atualmente esta sendo pesquisada e desenvolvida, na tentativa redução da excreção destes oocistos por este hospedeiro quando infectado, é a vacina contra o $C$. parvum, com a finalidade de produção de um colostro hiperimune capaz de minimizar a eliminação dos oocistos por estes bezerros (Mark, 2004).

Davis et al. (2010) estudaram o efeito da vacina $C$. parvum rCP15/60 em vacas, esta vacina foi desenvolvida com a finalidade de atribuir uma proteção passiva para os bezerros contra a criptosporidiose. Neste estudo foram estudados dois grupos de vacas, um de 20 vacas prenhes imunizadas com uma proteína recombinante de $C$. parvum, e um grupo controle de 20 vacas não imunizadas. Como resultado 
as vacas vacinadas apresentaram uma titulação de anticorpos significativamente superior as vacas do grupo controle, o mesmo foi observado no colostro destas vacas, deste modo a vacina pode conferir uma proteção passiva para bezerros contra a criptosporidiose.

\section{Alternativas terapêuticas:}

Mesmo com o avanço da medicina, ainda não há um tratamento especifico para criptosporidiose. (Ashbolt, 2004). Resultados mais expressivos entre os mamíferos terrestres foram alcançados por meio da administração de paramomicina e nitazoxanide, sendo o primeiro um antimicrobiano que reduz a excreção de oocitos (Steiner et al., 1997) e o segundo um antiprotozoário (Abaza et al., 1998). Além destes fármacos o suporte nutricional é recomendado com parte do tratamento (Steiner et al., 1997).

A paramomicina trata-se de um antibiótico aminoglicosídeo que possui efeitos de nefrotoxicidade e neurotoxidade, com efeito limitado para as parasitoses entéricas (Tavares, 1996). Hewitt et al. (2000), estudaram paramomicina em pacientes HIV soropositivos, e observaram que este fármaco não mostrou ser eficaz para 0 tratamento da enterite por Cryptosporidium sp, pois não notaram diferença estatística significativa entre o grupo placebo e o grupo que recebeu 0 fármaco.

O nitazoxanide foi estudado no controle da criptosporidiose por Rossingnol et al. (1998), que verificaram que sua ação foi de 63 a $67 \%$ de reposta satisfatória em indivíduos imunocomprometidos. Amadi et al. (2002) estudaram o efeito do nitazoxanide em crianças com criptosporidiose, e observaram apenas uma resposta satisfatória em $56 \%$ das crianças soronegativas para o HIV, em crianças soropositivas para o HIV, não foi observado benefício com a utilização do nitazoxanide.

\section{CONCLUSÃO}

Fatores como: (1) a inexistência de uma medida terapêutica adequada; (2) condutas profiláticas não totalmente seguras; (3) a contaminação do ambiente, principalmente da água, por oocistos excretados em grandes quantidades por hospedeiros infectados; (4) a grande facilidade de transmissão e de adquirir a infecção; (5) e a resistência da forma infectante do protozoário; permitem a ocorrência de surtos do criptosporidiose na população humana, especialmente pela veiculação hídrica.

Mesmo com o avanço em pesquisas, estudos sobre este coccídeo devem continuar a ser realizados com o intuito de se desenvolver medidas terapêuticas e profiláticas mais eficazes no seu controle, e principalmente quanto a diminuição da eliminação de oocistos pelo hospedeiro, minimizando a contaminação ambiental, reduzindo a possibilidade de novos surtos na população humana.

\section{REFERÊNCIAS}

ABAZA, H.; EL-ZAYADI, A. R.; KABIL, S. M.; RISK, $H$. Nitazoxanide in the treatment of patients with intestinal protozoan and helminthic infections: a report n 546 patients in Egypt.

Therapeutic Research, v.59, n. 2, p. 116-121, 1998.

ALMEIDA, A. J.; OLIVEIRA, F. C.; TEIXEIRA, C. $\mathrm{S}$. Risco relativo da infecção por parasitos do gênero Cryptosporidium em Bezerros Bovinos no Norte do Estado do Rio de Janeiro, Brasil.

Revista Brasileira de Parasitologia

Veterinária, v. 17, n. 1, p. 243-248, 2008.

AMADI, B.; MWIYA, M.; MUSSUKU, J. et al. Effect of nitozoxanide on morbidity and mortality in Zambian children with cryptosporidiosis. The Lancet, v. 360, n. 2, p. 1375-1380, 2002.

ARAI, H. Y. ; FUKUDA, T.; HARA, Y. et al. Prevalence of Cryptosporidium infection among 
domestic cats in the Tokyo metropolitan district, Japan. Japanese Journal of Medical Science and Biology, v. 7, n. 43, p.7-14, 1990.

ARAÚJO, A. J. U. S.; GOMES, A. H. S.; ALMEIDAM M. E.; KANAMURA, H. Y. Detecção de Cryptosporidium meleagridis em amostras fecais em pacientes HIV positivos no Brasil. Revista Panamericana de Infectologia, v. 9, n. 2, p. 38-40, 2007.

ASHBOLT, N. J. Microbial contamination of drinking water and disease outcomes in developing regions. Toxicology, v. 198, n. 1-3; p. 229-238, 2004.

BOLTER-BITZER, J. I.; LEE, H.; TREVORS, J. T. Molecular targets for detection and immunotherapy in Cryptosporidium parvum. Biotechonology Advanced, v. 26, n. 1, p. 1344, 2007.

CACCIO, S. M.; THOMPSON, R. C. A.; McLAUCHLIN, J.; SMITH, H. V. Unrevelling Crypyosporidium and Giardia epidemiology. Trends in Parasitology, v. 21, n. 9, p. 430-437, 2005.

CAPUANO, M. D.; ROCHA, G. M. Ocorrência de parasitas com potencial zoonótico em fezes de cães coletadas em áreas públicas do município de Ribeirão Preto. Revista Brasileira de Epidemiologia, v. 9, n. 1, p. 81-86, 2006.

CARVALHO-ALMEIDA, T.; PINTO, P. L.; QUADROS, C. M.; KANAMURA, H. Y.; CASIMIRO, A. M. Detecção de Cryptosporidium $s p$. em fezes não diarréicas em crianças, em um escola de educação infantil de São Paulo, Brasil. Revista do Instituto de Medicina Tropical, v. 48, n. 01, p. 27-32, 2006.

CHACIN-BONILLA, L. Importance of the different species and genotypes of Cryptosporidium in public health, Investigación Clínica. v. 43, n. 3, p. 215, 2001.

CHALMERS, R. M.; DAVIES, A. P. Minireview: Clinical cryptosporidiosis., Experimental Parasitology, v. 214, p. 138-146, 2010.

CHIEFF, P. P.; SENS, A. D. Y.;

PASCHOALOTTI, M. A. et al. Infecção por Cryptosporidium parvum em renais crônicos submetidos a transplante ou hemodiálise.

Revista da Sociedade Brasileira de Medicina Tropical, v. 31, n. 4, p. 333-337, 1998.

COSTA, F.; LACIR, F.; SILVA, T. J. P. B.; KENNETH, S. Cryptosporidium spp. in food.
Higiene Alimentar, v. 20, n. 142, p. 58-63, 2006.

DAVIS, R.; BROWN, A.; BROMAN, D. D. Antibody responses following administration of Cryptosporidium parvum rCP15/60 vaccine to pregnant cattle. Veterinary Parasitology, v. 9, n. 13, 2010.

DENG, M.; PETERSON, R. P.; CLIVER, D. O. First findings of Cryptosporidium an Giardia in California sea lions (Zalophus californianus). Journal Parasitology, v. 86, n. 3 p. 490-494, 2000.

DIETZ, V. et al. Active, multisite, laboratorybased surveillance for Cryptosporidium parvum. Journal American Tropical Medicine, v. 62, n. 3, p. 368-372, 2000.

EDERLI, B. B.; EDERLI, N. B.; OLIVEIRA, F. C. et al. Fatores de risco associados à infecção por Cryptosporidium spp. em cães domiciliados na cidade de Campo dos Goytacazes, Estado do Rio de Janeiro, Brasil. Revista Brasileira de Parasitologia, v. 17, n. 1, p. 250-256, 2008.

FAYER, R.; LEWIS, E. J.; TROUT, J. M. et al. Cryptosporidium parvum in oysters from commercial harvesting sites in the Chesapeake bay. Emerging Infectious Diseases. v. 5, n. 5, p. 706-710, 1999.

FAYER, R.; MORGAN, U.; UPTON, S. J. Epidemiology of Cryptosporidium transmission, detection and identification. International Journal for Parasitology, v. 30, n. 1, p. 12-13, 2000.

FEITOSA, F. L. F.; SHIMAMURA, G. M.; ROBERTO, T. et al. Prevalência de criptosporidiose em bezerros na região de Araçatuba, estado de São Paulo, Brasil, Ciência Rural, v. 34, n. 1, p. 189-193, 2004.

FELTUS, D. C.; GIDDIBGS, W. C.; SCHNECK, $B$, L. et al. Evidence supporting zoonotic transmission of Cryptosporidium spp. In Wisconsin. Journal of Clininical Microbiology, v. 44, n. 12, p. 4303-4308, 2008.

FÉRES, F. C. Ocorrência e caracterização molecular de Cryptosporidium spp. em cordeiros na região de Araçatuba -SP, Avaliação da Transferência da Imunidade Passiva. 2009. Araçatuba, 70f. Dissertação (Mestrado em Ciências Animal) - Curso de Pósgraduação em Ciências Animal, Universidade Estadual Paulista, "Júlio de Mesquita Filho" campus Araçatuba-SP. 
FIGUEIREDO, H. C. P.; PEREIRA JUNIOR, D. J.; NOGUEIRA, R. B.; COSTA, P. R. S.

Excreção de oocistos de Cryptosporidium parvum em cães saudáveis das cidades de Lavras e Viçosa, Estado de Minas Gerais, Brasil. Ciência Rural, v.34, n. 5, p. 1625-1627, 2004.

FIÚZA, V. R. S.; COSENDEY, R. L. L.; OLIVEIRA, F. Criptosporidiose suína associada aos sistemas de produção no estado do rio de janeiro. Revista Brasileira de Medicina Veterinária, v. 17, n. 1, p. 224-229, 2008.

GARLIPP C. R.; BOTTINI, P. V.; TEIXEIRA, A. T. L. S. Importância do diagnóstico laboratorial da criptosporidiose humana e outras coccidioses. Revista do Instituto de Medicina Tropical de São Paulo, v. 37, n. 5, p. 467-469, 1995.

GELLETLIE, R.; STUART, J.; SOLTANPOOR, N. et al. Cryptosporidium assiated with school milk. The Lancet, v. 350, n. 9083, p. 1005-1006, 1997.

GONÇALVES, E. M. N.; SILVA, A. J.; EDUARDO, M. B. P. et al. P. Multilocus genotyping of Cryptosporidium hominis assiciated with diarrhea outbreak in a Day care unit in São Paulo. Clinics, v. 6, n. 2, p. 119-126, 2006.

GRACZYK, T. K.; EVANS, B. M.; ZIF, C. J.; KARREMAN, JH. J.; PATZ, J. A. Environmental and geographical factors contributing to watershed contamination with Cryptosporidium parvum oocysts. Environmental research, v. 82, n. 3, p. 263-271, 2000.

HEWITT, R.G.; YIANNOUTSOS, C.T.; HIGGS, E.S. et al. Paromomycin: No more effective than placebo for treatment of cryptosporidiosis in patients with advanced HIV infection. Clinical Infictious Disease v. 31, n. 4, p. 1084-1092, 2000.

HUBER, E.; BOMFIM, T. C. B.; GOMES, R. S. Comparação entre infecção por Cryptosporidium $s p$. e por Giárdia sp. em gatos sob dois sistemas de criação. Revista Brasileira de Parasitologia Veterinária, v. 11, n. 1, p. 7-12, 2002.

HUGHES-HANKS, J. M.; RICKARD, L. G.; PANUSKA, C. et al. Prevalence of Cryptosporidium spp. and Giardia spp in five marine species. The Journal of Parasitology, v. 91 , n. 5, p. 1255-1228, 2005.

JOHNSON, D. C.; REYNOLDS, K. A.; GERBA, C. P. et al. Detection of Giardia and
Cryptosporidium genotypes in marine waters. Water Science and Technology. v. 31, n. 5-6, p. 439-442, 1995.

JOHNSON, J.; BUDDLE, R.; REID, S. et al. Prevalence of Cryptosporidium genotypes in pre and post-weaned pigs in Australia.

Experimental Parasitology. v. 119, n. 3, p. 418-421, 2008.

KUCZYNSKA, E.; SHELTON, D. R. Method for detection and enumeration of Cryptosporidium parvum oocysts in feces, manures and soils. Applied and Environmental Microbiology, v. 67, n. 7, p. 2820-2826, 1999.

LEAV, B. A.; MACHAY, M.; WARD, H. D. Cryptosporidium species: new insights and old challenges. Clinical Infectious Diseases. v. 36, n. 7, p. 906-908, 2003.

LUCCA, P.; GASPARI, E. N.; BOZZOLI, L. M. et al. Molecular characterization of Cryptosporidium spp. from HIV infectec patients from urban area of Brazil. Revista do Instituto de Medicina Tropical, v. 6, n. 51, p. 341-343, 2009.

MACHADO, E. C. L.; STANFORD, E. H. L.; SOARES, N. L. et al. Ocorrência de oocistos de Cryptosporidium spp. em água superficiais na região metropolitana de Recife/PE, Arquivo Brasileiro de Medicina Veterinária e Zootecnia, v. 61, n. 6, p. 1459-1462, 2009.

MARK, C. J. Present and future control of Crytptosporidium in humans and animals. Expert Review of Vaccines. v. 3, n. 6, p. 669671, 2004.

MATOS, D. J. Ocorrência de Cryptosporidium spp. (Tyzzer, 1907) e Giárdia spp. (Kunstler, 1982) em leitões ao desmame. 2009.

Araçatuba, 75f. Dissertação (Mestrado em Ciência Animal) - Curso de Pós-graduação em ciências animal Universidade Estadual Paulista, "Júlio de Mesquita Filho" campus Araçatuba-SP.

MILLARD, P. S.; GENSHEIMER, K. F.; ADDISS, D. G. et al. An outbreak of criptospodiriosis from fresh-pressed apple cider, Journal of the American Medicine Association, v. 272, n. 10, p. 1592-1196, 1994.

MOITINHO, M.; LUZ, R.; MARTINS, M. R. Ocorrência de Cryptosporidium sp. em fezes diarréicas no Município de Maringá-PR. Revista Brasileira de Analise Clínica, v. 29, n. 3. p. 168-170, 1997. 
MONGE, R.; CHINCHILLA, M. Presence of Cryptosporidium oocysts in fresh vegetables. Journal of Food Protection, v. 59, n. 2, p. 202 203, 2003.

MOURA, A. B.; TEIXEIRA, E. B.; SOUZA, A. P. et al. Cryptosporidium spp. em cães domiciliados da cidade de Lages, SC. Revista de Ciências Agroveterinárias, v.8, n. 2. p. 173178, 2009.

MTAMBO, M. M.; NASH, A. S.; BLEWETT, D. A. et al.Cryptosporidium infection in cats:

prevalence of infection in domestic and feral cats in the Glasgow area. Veterinary Research, v. 129, n. 23, p. 502-504, 1991.

NASCIMENTO, R. C.; CAVALCANTI, I. M. F.; IRMÃO, J. I.; ROCHA, J. S. Presença de Cryptosporidium spp. em crianças com diarréia aguda em um creche pública de Recife, Estado de Pernambuco. Revista da Sociedade Brasileira de Medicina Tropical, v. 43, n. 2, p. 175-178, 2009.

OLIVEIRA-SILVA, M. B.; OLIVEIRA, L. R.; RESENDE, J. C. P.; et al. Perfil sazonal e nível de linfócitos CD4+ na ocorrência de criptosporidiose e cistoisosporidiose em pacientes HIV/AIDS na região do Triângulo Mineiro, Brasil. Revista Sociedade Brasileira de Medicina Tropical, v. 40, n. 5, p. 542-515, 2007.

OLSON, M. E.; APPELBEE, A.; MEASURES, L. Giardia duodenalis and Cryptosporidium parvum infections in pinnipeds. Zoonotics protozoa in the marine environment: a threat to aquatic mammals and public health. Veterinary Parasitology, v. 125, p. 131-135, 2004.

ORTEGA, Y. R.; SHEEHY, R. R.; CAMA, V. A. et al. Restriction fragment length polymorphism analysis of Cryptosporidium parvum isolates of bovine and human origin. Journal of

Protozoology. v. 38, n. 6. p. 40S-41S, 1991.

OSHIRO, E. T.; DORVAL, M. E. C.; NUNES, V. L. B. et al.Prevalência do Cryptosporidium parvum em crianças abaixo de 5 anos, residentes na zona urbana de Campo Grande, MS, Brasil. Revista da Sociedade Brasileira de Medicina Tropical, v. 33, n. 3, p. 277-280, 2000.

OVERGAAUW, P A. W.; ZUTPHEN, L. V.; HOEK, D. et al. Zoonotic parasites is fecal samples and fur from dogs and cats in The Netheriands. Veterinary Parasitology, v. 13, n. 1, p. 115-122, 2009.
PALMER, C. R. ROBERTSON, I. D.; TRAUB, R. J.; DEVLIN, G. et al. Determining the zoonotic significance of Giardia and Cryptosporidium in Australian dogs and cats. Veterinary

Parasitology, v. 154, n. 1-2, p. 142-147, 2008.

PEREIRA, M. G. C.; ATWILL, E. R.; BARBOSA, A. P. et al. Intra-familial and extra-familial risk factors associated with Cryptosporidium parvum infection among children hospitalized for diarrhea in Goiânia, Goiás, Brazil, The American Journal of Tropical Medicine and Hygiene, v. 66, n. 6, p. 787-793, 2002.

PLUTZER, J. KARANIS, P. Genetic polymorphism in Cryptosporidium species: in update. Veterinary Parasitology, v. 165, n. 3-4, p.187-199, 2009

ROSSIT, A. R. B.; ALMEIDA, M. T.; NOGUEIRA, C. A. et al. Bacterial yeast, parasitic, and viral enteropathogens in HIV-infected children from São Paulo State. Southeastem Brasil,

Diagnostic Microbiology Infectious Disease, v. 57, n. 1, p. 59-66, 2007.

ROSSIGNOL, J. F.; HIDALGO, H.;

FEREGRINO, M. et al. A double-'blind' placebocontrolled study of nitazoxanide in the treatment of cryptosporidial diarrhoea in AIDS patients in Mexico. Transactions of the Royal Society of Tropical Medicine and Hygiene, v.92, n. 6, p. 663-666, 1998.

SILVA, G. A. P.; MAGALHÃES, M.; OLIVEIRA, A. M.; MELO, V. M. Diarréia aguda do lactante associada ao Cryptosporidium sp., na cidade de Recife. Jornal de Pediatria, v.70, n. 1, p. 44-46, 1994.

SPÓSITO-FILHA, E.; OLIVEIRA, S. M.

Criptosporidiose Divulgação Técnica. Instituto

Biológico, v. 71, n. 1, p. 17-19, 2009.

STEINER, T. S. THIELMAN, N. M.;

GUERRANT, R. L. Protozoal agents: what are the dangers for the public water supply? Annual Review Medicine, v. 48, p. 329-340, 1997.

SUNNOTEL, G.; LOWER, C. J.; MOORE, J. E. et al. Cryptosporidium. Letters in applied microbiology, v. 43, n. 1, p. 7-16, 2006.

SMITH A.; REACHER, M.; SMERDIG, W. et al. Outbreaks of waterborne infectious intestinal diseases in England and Wales, 1992-2003. Epidemiology and Infection. v. 134, n. 6, p. 1141-1149, 2008. 
SMITH, HV, NICHOLS RAB. Cryptosporidium: detection in water and food. Experimental of Parasitology, v. 124, n. 6, p. 61-79, 2010.

TAVARES, W. Manual de antibióticos e quimioterápicos antiinfecciosos, 2 ed. São Paulo: Atheneu, 1996, 626 p.

TEMBUE, A. A. M.; ALVES, L. C.; BORGES, J. C. G. et al. Ocorrência de Cryptosporidium spp. em ovinos no município de Ibimirim, estado de Pernambuco, Ciência Veterinária Tropical, v. 9, n. 1, p. 41-43, 2006.

THOMPSON, R. C. A.; PALMER, C. S.;

O'HANDLEY, R. The public health ad clinical significance of Giardia and Cryptosporidium in domestic animals. The Journal Veterinary, v. 177, n. 1, p. 18-25, 2008. 